\title{
Respostas metabólicas ao treinamento de força: uma ênfase no dispêndio energético
}

\author{
Metabolic responses to strength training: an emphasis \\ on energy expenditure
}

1 Universidade Federal do Rio Grande do Sul. Escola de Educação Física. Laboratório de Pesquisa do Exercício. Porto Alegre, RS. Brasil.

2 Hospital de Clínicas de Porto Alegre. Porto Alegre. RS. Brasil

Recebido em 15/12/09 Revisado em 24/06/10 Aprovado em 21/10/10
Resumo - O presente estudo revisa os resultados encontrados, até então, na literatura, relativos ao dispêndio energético (DE) no treino de força (TF). Para tanto, foi realizado um levantamento bibliográfico nas bases de dados Medline e Sport Discus, utilizando as palavras-chave: strength training, energy expenditure e excess post-exercise oxygen consumption (EPOC). Os estudos analisados referem que protocolos de TF que envolvam exercícios para grandes grupos musculares, com grandes intensidades e volumes de treinamento, e menores intervalos de recuperação entre séries e exercícios são os que apresentam maior impacto metabólico na sessão de treino. Essas mesmas variáveis também demonstram interferir na magnitude e na duração do EPOC.

Palavras-chave: Treinamento de força; Dispêndio energético; EPOC.

Abstract - The present study reviews the results reported in the literature regarding energy expenditure in strength training (ST). For this purpose, a search was conducted in the Medline and Sport Discus databases using the following key words: strength training, energy expenditure, and excess post-exercise oxygen consumption (EPOC). The studies analyzed report that ST protocols involving exercises for the major muscle groups characterized by high-intensity and high-volume training and low resting intervals between sets and exercises have a greater metabolic impact on ST. These variables also interfere with the magnitude and duration of EPOC.

Key words: Strength training; Energy expenditure; EPOC. 


\section{INTRODUÇÃO}

Nos últimos anos, o treino de força (TF) assumiu uma importância significativa em programas de intervenção direcionados a indivíduos com excesso de peso e/ou obesos. Os principais argumentos que justificam a sua proposição são o incremento da massa livre de gordura (MLG) e o consequente incremento das taxas metabólicas de repouso e diária (24-h), o qual repercute decisivamente na balança energética diária ${ }^{1,2}$.

No entanto, um dos pontos negativos associados ao TF relativamente à sua eficiência em termos de composição corporal é o reduzido impacto metabólico promovido numa sessão de treino desta natureza, que parece apresentar um DE inferior ao de modalidades aeróbias com intensidades moderadas a altas, fato este já referido por alguns investigadores ${ }^{3}$.

O dispêndio energético (DE) no TF parece depender da manipulação de inúmeras variáveis tais como: volume e intensidade administrados ${ }^{4}$, intervalo de recuperação entre séries e exercícios ${ }^{5}$ e velocidade de execução, além do método de treinamento utilizado. Entretanto, a literatura carece de trabalhos que esclareçam a real influência dessas variáveis no DE, durante e após sessões de TF. Sendo assim, essa revisão pretende salientar as principais formas de mensuração do DE no exercício físico, bem como analisar a influência que as diferentes formas de organização de uma sessão de TF exercem sobre o DE ocorrido durante e após esta sessão.

Para a elaboração deste trabalho, foi realizada uma busca bibliográfica nas bases de dados Medline e Sport Discus, utilizando as palavras-chave: strength training, energy expenditure, excess post-exercise oxygen consumption (EPOC).

\section{Dispêndio Energético no Exercício Físico}

O DE resulta da transferência de energia e consiste em produção de calor. Esta produção de calor tem servido como medida padrão para a interpretação do $\mathrm{DE}^{7}$. Em função das dificuldades na mensuração da perda de calor, o consumo de oxigênio $\left(\mathrm{VO}_{2}\right)$ constitui-se no método mais utilizado para estimar a produção de calor e, consequentemente, o $\mathrm{DE}$.

A calorimetria indireta tem sido utilizada como o método de estimativa do consumo de energia, quer em condições de repouso quer na prática do exercício físico ${ }^{1,8}$. A partir deste método, estima-se a quantidade total de energia gasta, utilizando-se para tal a quantidade de oxigênio consumido na oxidação dos substratos energéticos e o gás carbô- nico que é eliminado pela respiração quando são observadas condições de equilíbrio metabólico. Nestas condições, a calorimetria indireta é considerada um método prático para identificar a natureza e a quantidade dos substratos energéticos oxidados, bem como o total de energia gasta ${ }^{9}$.

\section{Dispêndio Energético Anaeróbio}

A mensuração do $\mathrm{VO}_{2}$ durante exercícios de força representa, parcialmente, o DE, neste tipo de exercício, subestimando o DE total decorrente desse tipo de exercício ${ }^{7,10}$. A oclusão do fluxo sanguíneo durante a contração muscular, a manobra de Valsalva, a presença do déficit de oxigênio e a ausência de um estado fisiológico estável revelam a incapacidade de, por meio do $\mathrm{VO}_{2}$, ser quantificado o DE durante a realização de exercícios de força. Atualmente, parece não haver dúvidas sobre a relevância do metabolismo anaeróbio no $\mathrm{DE}$ total em exercícios de força $a^{7,10,11}$.

A mensuração do DE anaeróbio tem sido mensurada a partir de: 1) biópsia muscular de metabólitos anaeróbios somado ao $\mathrm{VO}_{2}$ em exercício; 2) $\mathrm{VO}_{2}$ durante o exercício somado ao débito de oxigênio; ou 3) mensuração glicolítica/lactato somado ao $\mathrm{VO}_{2}$ do exercício e ao $\mathrm{EPOC}^{11}$. Segundo Bangsbo et al..$^{12}$, deve-se excluir 20\% do EPOC para a quantificação do DE total de uma sessão de exercícios de força ou de natureza anaeróbia, já que esta porcentagem incluiria a ressíntese de ATP-CP do metabolismo anaeróbio aláctico, o que já estaria incluído no consumo de oxigênio durante a passagem do período de repouso ao exercício (déficit de oxigênio).

Estimativas da concentração de lactato muscular pós-exercício de força têm mostrado contribuições significativas no DE total. Mensurações das concentrações de lactato (mmol) são convertidas em valores equivalentes de oxigênio. Tem sido proposto que, para cada milimole de lactato seja computado $3 \mathrm{ml} \mathrm{de} \mathrm{O}_{2}$ por quilograma de massa corporal $^{7}$, e posteriormente, convertido para quilocalorias a partir da relação: $5.05 \mathrm{kcal}$ para cada litro de oxigênio ${ }^{13}$.

Estas contribuições podem variar de acordo com o tipo de treinamento utilizado, sendo que poucos estudos têm mensurado esta contribuição ${ }^{8,14,15}$. Scott ${ }^{7}$ verificou a contribuição do lactato sanguíneo em protocolos de 2 séries de 3 exercícios de força (flexão de cotovelo, supino e leg press), em intensidades diferentes $(60 \%$ e $80 \%$ de 1 repetição máxima - 1RM). Na intensidade de 60\% de 1RM, em que as séries foram realizadas até a fadiga voluntária, foi observado um maior DE decorrente 
de maiores níveis de concentração de lactato pós-exercício, o que foi associado à maior demanda anaeróbia do exercício.

Além da intensidade e do número de repetições, as concentrações de lactato também estão associadas ao volume de treinamento e à velocidade de execução das repetições. Hunter et $\mathrm{al}^{8}{ }^{8}$ mostraram que o TF com velocidade tradicional decorre em maior contribuição anaeróbia ao DE total, comparado à velocidade super-lenta, e têm sido reportadas maiores concentrações de lactato após 3 séries de TF $(10.2 \pm 0.89)$ comparado a protocolos de série única $\left(7.9 \pm 0.69 \mathrm{mmol} \cdot \mathrm{L}^{-1} \cdot \mathrm{min}^{-1}\right)^{15}$. Essas diferenças parecem ocorrer como consequência do efeito cumulativo observado em séries múltiplas. Sendo assim, deve-se considerar que a omissão da contribuição do metabolismo anaeróbio pode levar a um erro considerável na estimativa do DE total decorrente de exercícios de força, principalmente, quando o componente anaeróbio destes exercícios for elevado.

\section{Dispêndio Energético e Treinamento de Força}

$\mathrm{Na}$ maioria dos estudos relativos ao $\mathrm{TF}$, foi utilizado o $\mathrm{VO}_{2}$ para a estimativa do $\mathrm{DE}$, sendo que a determinação da taxa de troca respiratória (RER) não pode ser utilizada para quantificar a proporção dos principais substratos oxidados (carboidratos e gorduras) neste tipo de exercício, pelo fato da inexistência de estado estável fisiológico ${ }^{16}$.

Wilmore et al. ${ }^{7}$ realizaram um dos primeiros estudos para quantificar a demanda metabólica em resposta a um protocolo de treino em circuito (3 séries a 40\% de 1RM), em homens e mulheres, e observaram um DE líquido de $130.6 \pm 34.5$ e $95.1 \pm 18.4 \mathrm{kcal}$, respectivamente. Melanson et al..$^{18}$, ao aplicarem o mesmo tipo de treinamento em homens jovens, observaram um DE líquido de $322 \pm 19 \mathrm{kcal}$, sendo este valor maior do que o observado no estudo anterior devido ao maior volume (4 séries) e intensidade (70\% de 1RM) utilizados.

Estudos têm apresentado valores de DE líquido próximos de 6 a $9 \mathrm{kcal}$ por minuto ${ }^{6,17,18} \mathrm{em}$ resposta ao treino em circuito, sendo que protocolos com 15-18 repetições em intensidades de, aproximadamente, $40 \%$ de $1 \mathrm{RM}$ e intervalos de recuperação de 15 segundos entre as séries, parecem incrementar de forma mais significativa o DE.

Outros métodos de TF parecem também ser efetivos no incremento do DE. O TF tradicional, por exemplo, intensidades entre $60-80 \%$ de $1 \mathrm{RM}$, 8 a 10 repetições por série, e $2-3$ séries com intervalos entre 1-2 minutos entre elas ${ }^{8,19,20}$, parecem promover um significativo impacto metabólico.
A maioria dos estudos relativos ao DE no TF utilizaram sessões de treino de diferentes configurações (intensidade, volume, número de séries, intervalos de recuperação, etc.), o que dificulta a comparação dos resultados destes estudos. Assim, a apresentação de valores relativos ao tempo (kcal. $\min ^{-1}$ ) facilita tal comparação.

\section{Volume de Treinamento}

Phillips e Ziuraitis ${ }^{13}$ mensuraram o DE de homens e mulheres jovens em um protocolo de 1 série de 8 exercícios e $15 \mathrm{RM}$. O protocolo apresentou um custo calórico de $5.63 \pm 0.7$ e $3.4 \pm 0.5$ kcal.min ${ }^{-1}$ para homens e mulheres, respectivamente. $\mathrm{O}$ mesmo protocolo foi utilizado em idosos pelos mesmos investigadore ${ }^{21}$ e o custo metabólico foi de $3.5 \pm 0.6 \mathrm{e}$ $2.9 \pm 0.7 \mathrm{kcal} . \mathrm{min}^{-1}$ para homens e mulheres, respectivamente. $\mathrm{O} \mathrm{DE}$ absoluto dos protocolos foi maior em homens, comparado às mulheres, em ambos os estudos ${ }^{13,21}$, o que parece ter sido decorrente do menor peso corporal, da TMR mais reduzida e da menor carga utilizada nos exercícios de força pelas mulheres e idosos.

Morgan et al. ${ }^{4}$ avaliaram o DE em 2 protocolos com intensidade e volume diferentes: 1) 2 séries de 8 repetições com intensidade de $100 \%$ de $8 \mathrm{RM} ; 2$ ) 2 séries de 15 repetições com intensidade de $85 \%$ de $8 \mathrm{RM}$. O DE foi de $2.8 \pm 1.5$ e $2.5 \pm 0.7 \mathrm{kcal} . \mathrm{min}^{-1}$ para homens e mulheres, respectivamente, no protocolo $1 \mathrm{e}$, ainda que não tenha sido observada diferença entre os sexos em termos temporais, quando relativo à massa corporal magra, este foi superior nas mulheres $(100 \% 8 \mathrm{RM}=6.3 \pm 1.9,85 \% 8 \mathrm{RM}=4.7 \pm 1.0$ $\left.\mathrm{kcal} \cdot \mathrm{Kg}_{\mathrm{MCM}}{ }^{-1} \cdot \mathrm{min}^{-1}\right)$, comparado aos homens $(100 \%$ $8 \mathrm{RM}=3.4 \pm 0.7,85 \% 8 \mathrm{RM}=2.3 \pm 0.8 \mathrm{kcal} \cdot \mathrm{Kg}_{\mathrm{MCM}}{ }^{-1}$. $\left.\mathrm{min}^{-1}, \mathrm{p}<0.02\right)$. Tais resultados sugerem que mulheres consomem mais oxigênio, em termos relativos, do que homens, em diferentes intensidades. Estes mesmos autores ${ }^{(4)}$ ressaltam que mulheres utilizam mais o metabolismo aeróbio e, consequentemente, transferem mais energia via metabolismo aeróbio do que os homens, quando realizam contrações musculares com intensidades superiores a 50\% de uma contração voluntária máxima (CVM). Kent-Braun et al. ${ }^{22}$ têm sugerido que isso se deve, em parte, a maior atividade de enzimas aeróbias e menor atividade de enzimas glicolíticas em mulheres do que em homens.

Haddock e Wilkin ${ }^{15}$ compararam o DE em protocolo de série única e de séries múltiplas de exercícios em mulheres pré-menopausicas. O DE líquido total foi maior em séries múltiplas $(158 \pm 10.5)$ do que em única $(56.15 \pm 3.2 \mathrm{kcal})$, sendo que os resultados 
são invertidos quando o $\mathrm{DE}$ é relativo ao tempo de execução de exercício ( 3 séries $=2.51 \pm 0.17$, e 1 série $\left.=2.61 \pm 0.13 \mathrm{kcal} \cdot \mathrm{min}^{-1}\right)$. Estes resultados não são justificados pelos autores.

Binzen et al. ${ }^{23}$ utilizaram a mesma população de Haddock e Wilkin ${ }^{15}$ ao mensurar o impacto metabólico de 3 séries de 10 RM, em 10 exercícios. Os resultados apresentaram um DE líquido de $157 \mathrm{kcal}$ (3.49 kcal.min ${ }^{-1}$ ). A diferença no custo calórico por minuto entre os dois estudos ${ }^{15,23}$ pode ter ocorrido devido às diferenças na composição corporal, nível de experiência em TF ou até mesmo, em razão do menor período de recuperação proposto por Binzen et al. ${ }^{23}$ (60 seg.versus 90 seg.).

\section{Intensidade de Treinamento}

Podem ser incluídas, além do intervalo de recuperação, outras variáveis de treinamento relacionadas ao TF, tais como: velocidade de execução do movimento e diferentes tipos de resistência empregada, que serão discutidas a seguir.

\section{Intervalo de Recuperação}

Ratamess et al..$^{5}$ compararam as respostas metabólicas de 10 diferentes protocolos de exercício com intensidades de $75 \%$ e $85 \%$ de $1 \mathrm{RM}$, para $10 \mathrm{RM}$ e $5 \mathrm{RM}$, respectivamente, utilizando intervalos de 30seg., 1, 2, 3 e 5 min, e observaram maior $\mathrm{VO}_{2}$ nos dois menores intervalos. Relativamente à intensidade, o protocolo de 10RM produziu maior custo metabólico comparado ao de 5RM $(6.0 \pm 1.3 \mathrm{kcal}$. min $^{-1}$ versus $5.7 \pm 1.2 \mathrm{kcal} . \mathrm{min}^{-1}$ ), ao longo de todos os intervalos de recuperação. Da mesma forma, Haltom et al. ${ }^{6}$ compararam, em homens, 2 circuitos com intervalos de 20 e 60 seg., e observaram um DE maior no circuito de menor intervalo de recuperação (8.58 e $6.56 \mathrm{kcal} \cdot \mathrm{min}^{-1}$, respectivamente). Estes resultados sugerem que menores intervalos de recuperação entre séries e exercícios aumentam a intensidade da sessão de treinamento, provocando maiores valores de $\mathrm{DE}$.

\section{Velocidade de Execução}

Hunter et al. ${ }^{8}$ compararam as respostas metabólicas em TF com velocidades super-lenta (TSL) e tradicional (TT), tendo observado um maior DE líquido no protocolo TT $\left(\mathrm{TSL}=2.56\right.$, e TT= 4 kcal. $\mathrm{min}^{-1}$ ). Esta diferença significativa no DE é influenciada pelo trabalho total realizado e pela intensidade relativa do exercício que, neste caso, foi 2.6 vezes maior no TT ( $65 \%$ versus $25 \%$ de $1 \mathrm{RM})$. Além disso, o trabalho total realizado no TT foi cerca de 4 vezes o realizado no TSL.
O TF realizado com velocidade lenta inclui um maior componente isométrico, o qual já foi referido por McArdle e Foglia ${ }^{19}$. Neste estudo ${ }^{19}$, os autores compararam o custo energético entre TF tradicional (TFT) e isométrico (TFI). O TFT foi realizado com 8 repetições máximas, enquanto o TFI foi realizado com 6 segundos de contração máxima. $\mathrm{O} \mathrm{VO} 2$ decorrente do TFT foi duas vezes maior do que do TFI, o que pode ter ocorrido em função do menor tempo de duração e pelo fato dos sujeitos realizarem o bloqueio respiratório durante as contrações isométricas, produzindo um menor consumo de oxigênio e consequentemente, menor $\mathrm{DE}$ em exercícios isométricos comparado aos tradicionais.

\section{Diferentes Tipos de Resistência}

Em um dos poucos estudos em que foram utilizados equipamentos com resistência hidráulica, nos quais é realizada somente a fase concêntrica do movimento, Ballor et al. ${ }^{24}$ observaram um $\mathrm{VO}_{2}$ de $1.93 \pm 0.12$ L. min $^{-1}$ durante o protocolo, o que corresponde a um DE de $9.75 \mathrm{kcal} \cdot \mathrm{min}^{-1}$, sendo estes valores superiores aos reportados nos estudos previamente citados, aproximando-se apenas dos valores apresentados por Haltom et al..$^{6}\left(1.7 \mathrm{~L} . \mathrm{min}^{-1}\right)$, os quais justificam os resultados pela natureza dos exercícios.

\section{Consumo excessivo de oxigênio pós- exercício (EPOC) em Treinamento de Força}

O consumo excessivo de oxigênio pós-exercício (EPOC) é a elevação da taxa metabólica $\left(\mathrm{VO}_{2}\right.$ repouso ) acima dos níveis pré-exercício durante a recuperação $0^{25}$.

$\mathrm{O} \mathrm{VO}_{2}$ se mantém elevado, acima dos níveis de repouso, após vários tipos de exercícios, entre os quais a caminhada em esteira rolante, bicicleta ergometria, e treinamento de forçç ${ }^{5,6,14,15,19,20,23,26-33}$. A literatura sugere que exercícios de força realizados em intensidades acima de 70\% de 1RM apresentam maior magnitude de $\mathrm{EPOC}^{28}$. Diferentes níveis de EPOC podem ser observados de acordo com a organização das variáveis de treinamento nos diferentes protocolos de exercício.

\section{Volume de Treinamento}

Poucos estudos têm observado a diferença do EPOC entre protocolos de série única e séries múltiplas ${ }^{15,30}$. Murphy e Schwarzkop $\mathrm{f}^{30}$ mensuraram o EPOC entre protocolos de séries múltiplas (50\% 1RM), e série única ( $80 \%$ 1RM). Embora a intensidade relativa tenha sido maior no protocolo de série única, os resultados mostram que tanto a magnitude $(0.25 \mathrm{e}$ 
0.18 L.min ${ }^{-1}$, repectivamente) quanto a duração do EPOC (20 versus 15 minutos) foi superior em séries múltiplas comparado à série única. A carga utilizada no treinamento foi muito similar entre os dois protocolos. Entretanto, a intensidade absoluta no protocolo de séries múltiplas foi substancialmente maior comparado à série única. Estas diferenças em magnitude e duração do EPOC podem estar relacionadas pela relação trabalho:recuperação de 1:1 e 1:5, respectivamente. Maiores intervalos de recuperação irão permitir que o lactato, as concentrações de ATP-CP, e a temperatura corporal retornem parcialmente ou totalmente aos valores pré-exercício. Sendo assim, parece que a relação entre o intervalo de recuperação e o EPOC está inversamente relacionada, o que será explorado a seguir.

Haddock e Wilkin ${ }^{15}$ compararam em mulheres treinadas o EPOC decorrente de protocolos de mesma intensidade (8RM), porém diferentes volumes (1 e 3 séries). Neste caso, os resultados mostraram que o aumento do volume de exercício aumenta o DE durante o mesmo, porém não altera o EPOC avaliado por $120 \mathrm{~min}$ ( 1 série $=4.42$, e 3 séries $=$ 4.46 $\mathrm{L} \mathrm{O}_{2}$ ), o que não foi justificado pelos autores.

\section{Intensidade de Treinamento}

Olds et al. ${ }^{34}$ realizaram o primeiro trabalho com o objetivo de investigar a resposta ao EPOC entre protocolos de treino de força de igual volume e diferentes intensidades, não tendo sido observada nenhuma diferença significativa entre estes. No entanto, a diferença de intensidade (60\% e 75\% de 1RM) parece não ter sido suficientemente elevada para provocar diferenças nesta variável.

Thornton e Potteiger ${ }^{20}$ compararam o EPOC decorrente de dois protocolos de igual volume e diferentes intensidades: 1) 2 séries de 8 repetições a $85 \%$ de $8 \mathrm{RM}$ e, 2) 2 séries de 15 repetições a $45 \%$ de $8 \mathrm{RM}$. Os resultados mostraram que no período pós-exercício: 0-20, 45-60, e 105-120 minutos, os valores de EPOC foram significativamente maiores no protocolo de alta intensidade, o que parece ocorrer devido ao maior distúrbio metabólico durante o exercício, exigindo DE aumentado pós-exercício, a fim de restabelecer os processos fisiológicos.

No TF de alta intensidade são produzidas maiores magnitudes de frequência cardíaca (FC), concentração de lactato e sensação subjetiva de esforço (SSE), indicando maior estresse fisiológico. Este estresse é evidente na maior magnitude do EPOC, nos primeiros 20 minutos após o exercício, numa sessão de TF de alta intensidade ${ }^{20}$. A velocidade de execução do movimento e o intervalo de recuperação entre as séries e os exercícios também parecem influenciar a magnitude do EPOC.

\section{Velocidade de execução}

Hunter et al. ${ }^{8}$ foram um dos poucos investigadores a comparar as respostas metabólicas no TF com velocidade tradicional (TT) (0.9 segundos de fase concêntrica e 0.8 segundos de fase excêntrica) e super-lenta (TSL) (10 segundos concêntrica e 5 segundos excêntrica), e reportaram valores de EPOC superiores no TT $\left(8.2 \pm 2.0 \mathrm{~L} \mathrm{O}_{2}\right)$ comparado ao TSL $\left(6.7 \pm 1.7 \mathrm{~L} \mathrm{O}_{2}\right)$. Segundo os autores, esta diferença estaria associada ao trabalho total realizado e à intensidade relativa do exercício.

\section{Intervalo de recuperação}

O tempo de recuperação entre séries e repetições parece afetar as respostas metabólicas após sessões de $\mathrm{TF}$, sendo que a maioria dos estudos utilizou intervalos de 1 e 2 minutos ${ }^{14,20,23,26-28,30}$, com resultados variados.

Haltom et al. ${ }^{6}$ empregaram dois protocolos iguais de TF, porém com diferentes intervalos de recuperação (20 e 60 seg.), e observaram que o EPOC, avaliado durante 1 hora, foi significativamente maior no protocolo de 20 segundos de intervalo, comparado ao de 60 segundos $(0.53 \pm 0.04$ versus $0.49 \pm 0.032$ L.min $\left.{ }^{-1}\right)$. A maior diferença foi observada durante os 5 minutos iniciais de recuperação (maior componente rápido do EPOC).

Ratamess et al. ${ }^{5}$, ao mensurarem o EPOC em protocolos com intensidades de 75\% (10RM) e $85 \%$ de 1RM (5RM), e intervalos de 30 segundos, 1, 2, 3 e 5 minutos de duração, não observaram diferença significativa nos valores absolutos de EPOC $\left(10.3 \pm 3.5\right.$ vs. $\left.10.0 \pm 3.2 \mathrm{~L} \mathrm{O}_{2}\right)$ entre $5 \mathrm{RM} \mathrm{e}$ 10RM, respectivamente. Sendo assim, neste estudo, os intervalos de recuperação não tiveram efeito significativo no EPOC.

Quando todas as variáveis no TF se mantêm constantes e o intervalo de recuperação é alterado, maiores períodos de recuperação resultam em maior DE em exercício, enquanto que menores períodos de recuperação resultam em maiores valores de EPOC ${ }^{6}$, que estão associados a maior estresse fisiológico. Entretanto, um recente trabalho ${ }^{33}$ sugere que a ordem de execução dos exercícios para o mesmo grupo muscular (alternado por segmento vs. pré-exaustão) parece não interferir no EPOC. Os resultados desse estudo $^{33}$ mostram um EPOC elevado, uma vez que pouco ou nenhum intervalo foi administrado entre os exercícios, em ambos os métodos de treinamento, sem que diferenças fossem encontradas entre eles. 
Embora muitos fatores contribuam para o maior EPOC no TF, dois fatores em particular podem afetar de forma significativa este comportamento. Primeiro, perturbações hormonais, particularmente catecolaminas, cortisol e hormônio do crescimento $(\mathrm{GH})$, podem ser substanciais, especialmente, se o número de repetições por série for grande $(>5)$ e se o período de intervalo entre séries for $\leq 1$ minuto $^{22}$. Efeitos residuais de hormônios podem apresentar maior efeito sob o consumo energético da recuperação como resultado do TF. Segundo, também é possível que danos nos tecidos conjuntivos e contráteis e o estímulo de hipertrofia tecidual resultante do TF possam ser suficientes para contribuir com a maior necessidade energética durante o período de recuperação. Tem sido mostrado que os níveis de catecolaminas aumentam em resposta aguda aos exercícios de força, embora o treinamento não pareça alterar estas respostas ${ }^{35}$. Da mesma forma, os níveis de cortisol são aumentados mais expressivamente em resposta ao elevado número de repetições e baixa intensidade (10RM), comparado ao baixo número de repetições e elevada intensidade (5RM). Mais do que isso, o aumento do período de intervalo de 1 para 3 minutos reduziria a resposta do cortisol ${ }^{36}$. E, finalmente, as variáveis agudas do TF (repetições, intensidade e intervalo de recuperação) têm mostrado os mesmos efeitos sob o $\mathrm{GH}^{37}$.

\section{Treinamento de Força e Quociente Respiratório}

O quociente respiratório (RER) é a relação entre o dióxido de carbono produzido $\left(\mathrm{VCO}_{2}\right)$ e o volume de oxigênio consumido $\left(\mathrm{VO}_{2}\right)$. A partir desta relação $\left(\mathrm{VCO}_{2} / \mathrm{VO}_{2}\right)$, denominada taxa de troca respiratória, estima-se a porcentagem da contribuição dos carboidratos e das gorduras ao metabolismo energético durante o exercício. No entanto, para esta análise ser válida, há a necessidade da existência de condições fisiológicas em estado de equilíbrio.

No TF, por não haver estado de equilíbrio em razão do aumento abrupto da produção de $\mathrm{CO}_{2}$ por consequência da hiperventilação, o que ocasiona um $R E R$ não verdadeiro com valores acima de 1 , vários autores $5,6,8,20,27,30,32$ utilizam o $R E R$ não-protéico com o equivalente calórico de $5.05 \mathrm{kcal}$ por litro de $\mathrm{O}_{2}$ consumido para a estimativa do DE em TF. O mesmo ocorre durante o período de recuperação, em que a estimativa do DE a partir do equivalente calórico do RER pode ser feita somente após as concentrações de lactato retornarem aos valores pré-exercício, em razão da elevada produção de $\mathrm{CO}_{2}$ durante a oxidação do lactato ${ }^{23}$.
Estudos mostram que o RER permanece elevado acima dos níveis de repouso durante o $\mathrm{TF}^{18,24,26}$. A justificativa para os altos valores de RER encontrados durante o TF é a grande demanda ventilatória exigida neste tipo de exercício. Ballor et al..$^{24}$, ao avaliarem uma sessão de TF em circuito, observaram níveis elevados de RER $(0.99 \pm 0.01)$, que se assemelham aos encontrados por Melanson et al. ${ }^{18}$ e Braun et al. ${ }^{26}(1.02 \pm 0.01$ e $1.11 \pm 0.05$, respectivamente).

Imediatamente após o término do TF, os valores de RER mantêm-se elevados por curtos períodos de tempo ${ }^{24,27}$, apresentando uma redução do mesmo ao longo da recuperação $0^{15,23,24,27,31,32}$. Ballor et al. ${ }^{24}$ encontraram RER significativamente mais baixo do minuto 35-50 da recuperação, indicando maior taxa de oxidação de gorduras durante a recuperação do TF. Osterberg e Melby ${ }^{31}$ apresentaram um aumento de $62 \%$ na oxidação de gorduras, no dia seguinte após TF (pré $=0.841 \pm 0.02$, pós-exercício $=0.807 \pm 0.02, \mathrm{p}=.015)$. No entanto, os protocolos testados por Haddock e Wilkin ${ }^{15}$ foram os que apresentaram menores valores de RER, e consequentemente, maior oxidação de gorduras, durante a recuperação de TF de série única e séries múltiplas de 10 RM $(0.74 \pm 0.03$ e $0.75 \pm 0.04$, respectivamente), valores estes que se aproximam dos encontrados por Binzen e colaboradores ${ }^{(4)}(0.75 \pm 0.05)$ após TF a $70 \% 1 \mathrm{RM}$.

O desempenho no TF de alta intensidade é dependente do metabolismo anaeróbio de fosfocreatina e glicogênio para a produção de energia, levando a uma depleção dos estoques de glicogênio ${ }^{25}$, o que acaba por incrementar a taxa de oxidação de gorduras.

Relativamente a estudos longitudinais, Treuth et al. ${ }^{38}$, Lemmer et al. ${ }^{39}$ e Poehlman ${ }^{40}$ observaram alterações de RER em repouso após períodos de TF. Entre estes, Treuth et al. ${ }^{38}$ foram os únicos a mostrarem diferença significativa de RER após 16 semanas de TF. Os resultados mostraram redução significativa do RER em repouso após período de TF $(0.87 \pm 0.02$ vs. $0.81 \pm 0.02, \mathrm{p}<0.05)$, revelando significante aumento da oxidação de gorduras em 24-h ( $42 \pm 6$ vs. $81 \pm 7 \mathrm{~g} /$ dia, $\mathrm{p}<0.001$ ) e redução da oxidação de carboidratos $(180 \pm 14$ vs. $113 \pm 10 \mathrm{~g} / \mathrm{dia}, \mathrm{p}<0.001)$. No entanto, os estudos de Lemmer e colaboradores ${ }^{39}$ e Poehlman et al..$^{40}$ não mostraram diferença significativa após período de 24 semanas de TF.

\section{CONSIDERAÇÕES FINAIS}

Os estudos revisados sugerem que o DE em exercício e em recuperação são dependentes das variáveis de 
treinamento utilizadas (volume, intensidade, número de séries e repetições, velocidade de execução do movimento e intervalo de recuperação). Aumentos no DE em resposta ao TF são observados em exercícios que envolvem grandes grupos musculares, alta intensidade, grande volume de treinamento e menores intervalos de recuperação.

A magnitude e a duração do EPOC estão relacionadas ao grau de distúrbio à homeostase provocado pelo exercício. Intensidades de, no mínimo, 70\% de 1RM mostram ser o maior determinante da magnitude do EPOC. Além disso, intervalos curtos entre as séries e os exercícios parecem aumentar os níveis de EPOC.

\section{REFERÊNCIAS BIBLIOGRÁFICAS}

1. Ades PA, Savage PD, Brochu M, Tiscler MD, Lee NM, Poehlman ET. Resistance training increases total daily energy expenditure in disabled older women with coronary heart disease. J Appl Physiol 2005; 98(4):1280-5.

2. Melanson EL, Sharp TA, Seagle HM, Donahoo WT, Grunwald GK, Peters JC, et al. Twenty-four-hour metabolicresponses to resistance exercise in women. J Strength Cond Res 2005;19(1):61-6.

3. Laforgia J, Withers RT, Shipp NJ, Gore CJ. Comparison of energy expenditure elevations after submaximal and supramaximal running. J Appl Physiol 1997;82(2): 661-6.

4. Morgan B, Woodruff SJ, Tiidus PM. Aerobic energy expenditure during recreational weight training in females and males. J Sports Sci Med 2003;2(3):117-22.

5. Ratamess NA, Falvo MJ, Mangine GT, Hoffmann JR, Faigenbaum AD, Kang J. The effect of rest interval length on metabolic responce to the bench press exercise. Eur J Appl Physiol 2007;100(1):1-17.

6. Haltom RW, Kraemer RR, Sloan RA, Hebert EP, Frank $\mathrm{K}$, Tryniecki JL. Circuit weight training and its effects on excess postexercise oxygen consumption. Med Sci Sports and Exerc 1999;31(11):1613-8.

7. Scott CB. Contribution of Blood Lactate to the Energy Expenditure of Weight Training. J Strength Cond Res 2006; 20(2): 404-411.

8. Hunter GR, Seelhorst D, Snyder S. Comparison of metabolic and heart rate responses to super slow vs. traditional resistance training. J Strength Cond Res 2003;17(1):76-81.

9. Ferrannini E. The theoretical bases of indirect calorimetry: a review. Metabolism 1988;37(3):287-301.

10. Gaesser GA, Brooks GA. Metabolic bases of excess post-exercise oxygen consumption: a review. Med Sci Sports and Exerc 1984;16(1):29-43.

11. Scott CB, Kemp RB. Direct and indirect calorimetry of lactate oxidation: implications for whole-body energy expenditure. J Sports Sci 2005 23(1):15-19.

12. Bangsbo J, Gollnick PD, Graham TE, Juel C, Kiens B, Mizuno M, et al. Anaerobic Energy Production and O2
Deficit-Debt Relationship During Exhaustive Exercise in Humans. J Physiol 1990;422:539-59.

13. Phillips WT, Ziuraitis JR. Energy cost of single-set resistance training in older adults. J Strength Cond Res 2004;18(3):606-9.

14. Burleson MA, O’Bryant HJr, Stone MH, Collins MA, Triplett-McBride T. Effect of weight training exercise and treadmill exercise on post-exercise oxygen consumption. Med Sci Sports and Exerc 1998;30(4):518-22.

15. Haddock BL, Wilkin LD. Resistance training volume and post exercise energy expenditure. Int J Sports Med 2006;27(2):143-8

16. Gastin PB. Energy System Interaction and Relative Contribution During Maximal Exercise. Sports Med 2001;31(10):725-41.

17. Wilmore JH, Parr RB, Ward P, Vodak PA, Barstow TJ, Pipes TV, et al. Energy Cost of circuit weight training. Med Sci Sports 1978;10(2):75-8.

18. Melanson EL, Sharp TA, Seagle HM, Donahoo WT, Grunwald GK, Peters JC, et al. Resistance and aerobic exercise have similar effects on 24-h nutrient oxidation. Med Sci Sports and Exerc 2002; 34(11):1793-800.

19. McArdle WD, Foglia GF. Energy cost and cardiorespiratory stress of isometric and weight training exercises. J Sports Med 1969;9:23-30.

20. Thornton MK, Potteiger JA. Effects of resistance exercise bouts of different intensities but equal work on EPOC. Med Sci Sports and Exerc 2002;34(4):715-22.

21. Phillips WT, Ziuraitis JR. Energy cost of the ACSM single-set resistance training protocol. J Strength Cond Res 2003;17(2):350-5.

22. Kent-Braun JA, Ng AV, Doyle JW, Towse TF. Human skeletal muscle responses vary with age and gender during fatigue due to incremental isometric exercise. J Appl Physiol 2002;93(5):1813-23.

23. Binzen CA, Swan PD, Manore MM. Postexercise oxygen consumption and substrate use after resistance exercise in women. Med Sci Sports and Exerc 2001;33(6):932-8.

24. Ballor DL, Becque MD, Katch VL. Metabolic responses during hydraulic resistance exercise. Med Sci Sports and Exerc 1987;19(4):363-7.

25. Gaesser GA, Brooks GA. Metabolic bases of excess post-exercise oxygen consumption: a review. Med Sci Sports and Exerc 1984;16(1):29-43.

26. Braun WA, Hawthorne WE, Markofski MM. Acute EPOC response in women to circuit training and treadmill exercise of matched oxygen consumption. Eur J Appl Physiol 2005;94(5-6):500-4.

27. Crommett AD, Kinzey SJ. Excess postexercise oxygen consumption following acute aerobic and resistance exercise in women who are lean or obese. J Strength Cond Res 2004;18(3):410-5.

28. Drummond MJ, Vehrs PR, Schaalje GB, Parcell AC. Aerobic and resistance exercise sequence affects excess postexercise oxygen consumption. J Strength Cond Res 2005;19(2):332-7.

29. Melby C, Scholl C, Edwards G, Bullough R. Effect of acute resistance exercise on post-exercise energy 
expenditure and resting metabolic rate. J Appl Physiol 1993; 75(4):1847-53.

30. Murphy E, Schwarzkopf R. Effects of Standard Set and Circuit Weight Training on Excess Post-exercise Oxygen Consumption. J Appl Sport Sci Res 1992;6(2):88-91.

31. Osterberg KL, Melby CL. Effect of acute resistance exercise on postexercise oxygen consumption and resting metabolic rate in young women. Int J Sport Nut Exerc Met 2000;10(1):71-81.

32. Pinto RS. Adaptações metabólicas, cardio-respiratórias, neuromusculares e na composição corporal de mulheres pré-menopaúsicas e com excesso de peso em resposta ao treino físico sistemático. Tese de Doutorado. Lisboa 2007

33. Silva, FL, Brentano, MA, Kruel, LFM. Effects of Different Strength Training Methods on Postexercise Energetic Expenditure. J Strength Cond Res 2010;24(8):2255-60.

34. Olds TS, Abernathy PJ. Postexercise oxygen consumption following heavy and light resistance exercise. J Strength Cond Res 1993;7(3):147-52.

35. Kraemer WJ, Fry AC, Warren BJ, Stone MH, Fleck SJ, Kearney JTC. et al. Acute hormonal responses in elite junior weightlifters. Int J Sports Med 1992;13(2): 103-9.

36. Kraemer WJ, Fleck SJ, Dziados JE, Harman EA, Marchitelli LJ, Gordon SE, et al. Changes in hormonal concentrations after different heavy resistance exercise protocols in women. J Appl Physiol 1993;75(2):594-604.

37. Kraemer WJ, Gordon SE, Fleck SJ, Marchitelli LJ, Mello R, Dziados JE, et al. Endogenous anabolic hormonal and growth factor responses to heavy resis- tive exercise in males and females. Int J Sports Med 1991;12(2):228-35

38. Treuth MS, Hunter GR, Weinsier RL, Kell SH. Energy expenditure and substrate utilization in older women after strength training: 24-h calorimeter results. J Appl Physiol 1995;78(6):2140-6.

39. Lemmer JT, Ivey FM, Ryan AS, Martel GF, Hurlbut DE, Metter JE, et al. Effect of strength training on resting metabolic rate and physical activity: age and gender comparisons. Med Sci Sports and Exerc 2000;33(4):532-41.

40. Poehlman ET, Denino WF, Beckett T, Kinaman KA, Dionne IJ, Dvorak R, et al. Effects of endurance and resistance training on total daily energy expenditure in young women: a controlled randomized trial. J Clinl End Met 2002;87(3):1004-9.

\section{Endereço para correspondência}

Ronei Silveira Pinto

Laboratório de Pesquisa do Exercício, UFRGS, Rua Felizardo, 750

CEP: 90690-200. Porto Alegre, RS - Brasil

E-mail: ronei.pinto@ufrgs.br 